\title{
Dynamical Vertex Approximation for Nanoscopic Systems
}

\author{
A. Valli, ${ }^{1}$ G. Sangiovanni, ${ }^{1}$ O. Gunnarsson, ${ }^{2}$ A. Toschi, ${ }^{1}$ and K. Held ${ }^{1}$ \\ ${ }^{1}$ Institute of Solid State Physics, Vienna University of Technology, 1040 Vienna, Austria \\ ${ }^{2}$ Max-Planck-Institut für Festkörperforschung, 70569 Stuttgart, Germany
}

(Received 11 March 2010; published 16 June 2010)

\begin{abstract}
With an increasing complexity of nanoscopic systems and the modeling thereof, new theoretical tools are needed for a reliable calculation of complex systems with strong electronic correlations. To this end, we propose a new approach based on the recently introduced dynamical vertex approximation. We demonstrate its reliability already on the one-particle vertex (i.e., dynamical mean field theory) level by comparison with the exact solution. Modeling a quantum point contact with 110 atoms, we show that the contact becomes insulating already before entering the tunneling regime due to a local Mott-Hubbard transition occurring on the atoms which form the point contact.
\end{abstract}

PACS numbers: 71.27.+a, 71.10.Fd, 79.60.Jv

Introduction.-In recent years, we have seen tremendous experimental progress in the direction of man-made nanostructures. For example, with the wizardry of modern semiconductor technology quantum effects in quantum dots could be revealed [1-7]; in the area of molecular electronics transport through single molecules can now be studied [8-10]; and for magnetic storage technology nanoclusters of transition metals on surfaces become relevant [11]. In all three examples electronic correlations play a decisive role since the restriction to nanostructures brings the electrons close to each other so that their mutual Coulomb interaction becomes large (compared to their kinetic energy or tunneling rates). As a matter of fact, electronic correlations are not only genuine to nanostructures, but they also make them fascinating, both from the basic research point of view, with new physics occurring, and from the point of applications since strong correlations result in spectacular physical properties. An example is the Kondo physics which overcomes the Coulomb blockade and which has been observed in the conductance of quantum dots [2-5,7] as well as for small clusters and individual adatoms [12-15].

The theoretical modeling of strong correlations in nanostructures attached to some environment (bath) such as the source and drain electrode in case of the quantum dot or the surface for the transition metal cluster is hitherto based on generalizations of the Anderson impurity model (AIM) [5,7,16-18]. However, if one is not only dealing with a single or two "sites" (say the number of quantum dots), the numerical effort to solve the corresponding AIM becomes prohibitively expensive. More precisely, the effort grows exponentially with the number of sites for an exact $[19,20]$ or numerical renormalization group [18] treatment. This restricts these methods to $O(2)$ sites coupled to a bath. Related dynamical matrix renormalization group approaches [21] might allow for slightly larger systems but ultimately suffer from the same nonpolynomial problem, except for truly one-dimensional geometries. Potentially more efficient quantum Monte Carlo (QMC) methods [22] on the other hand exhibit a growing sign problem with increasing system size, restricting such methods to $O(10)$ sites. Hence a good theory for correlated nanosystems with even a few coupled nano-objects is presently missing. Such a theory is, however, mandatory since future technological applications will require the engineering of complex networks of such nano-objects-be it for a quantum computer or for a von Neuman computer based on such small structures so that quantum effects are no longer negligible.

On the other side, dynamical mean field theory (DMFT) [23-26] along with its cluster [22] and diagrammatic extensions such as the dynamical vertex approximation (DГA) [27] and the dual fermion approach [28] has been applied to strongly correlated electron systems with great success: on the model level, among others, the MottHubbard transition [24,25], magnetism [29], and kinks in strongly correlated systems [30-32] could be better understood or have even been discovered. Merging DMFT with density functional theory in the local density approximation (LDA) [33-36] turned out to be a breakthrough for the calculation of actual materials with strong correlations. By construction, these DMFT calculations are done in the thermodynamic limit, i.e., for a macroscopically large system.

There has been one attempt by Florens [37] to establish a nanoscopic version of DMFT. The idea behind Florens's nano-DMFT approach is the limit of a large number of neighbors with a central site in the middle, surrounded by many neighbors which in turn are coupled to many neighbors, etc. In such a geometry (e.g., of a Cayley-type tree), one gets a recursive method where the inner sites depend on their outer neighbors but not vice versa. Experimentally, however, such a geometry with more and more neighbors is hardly realizable, and the approach has been scarcely used in practice [38].

Here, we hence take another route based on the DГА concept of the locality of the fully irreducible $n$ particle vertex $\Gamma$. While the calculations in this first paper will be for $n=1$, i.e., on a DMFT-like level, we call the approach 
nano-DГA in the following - also to distinguish it from the aforementioned nano-DMFT. Below, we introduce the approach, validate its range of applicability against the exact numerical solution for system sizes where this is still possible, and demonstrate its potential by hands of calculations for a quantum point contact with 110 sites.

Method.-As pointed out in the introduction, we are interested in a nanoscopic system consisting of nanoobjects (sites) $i$ which are hybridized via $t_{i j}$, interacting by a Coulomb repulsion $U_{i}$ and coupled by $V_{i \nu k}$ to some noninteracting environment, see Fig. 1. The Hamiltonian hence reads

$$
\begin{aligned}
H= & \sum_{i j \sigma} t_{i j} c_{i \sigma}^{\dagger} c_{j \sigma}+\sum_{i} U_{i} c_{i \uparrow}^{\dagger} c_{i \uparrow} c_{i \downarrow}^{\dagger} c_{i \downarrow}+\sum_{i \nu k \sigma} V_{i \nu k} c_{i \sigma}^{\dagger} l_{\nu k \sigma} \\
& + \text { H.c. }+\sum_{\nu k \sigma} \epsilon_{\nu k} l_{\nu k \sigma}^{\dagger} l_{\nu k \sigma},
\end{aligned}
$$

where $c_{i \sigma}^{\dagger}\left(c_{i \sigma}\right)$ and $l_{\nu k \uparrow}^{\dagger}\left(l_{\nu k \uparrow}\right)$ denote the creation (annihilation) operators for an electron with spin $\sigma$ on site $i$ and in lead $\nu$ state $k$ with energy $\epsilon_{\nu k}$, respectively. While we consider a single band situation in the following, Hamiltonian (1) can easily be generalized to include orbital realism, leading to an additional orbital index in the second

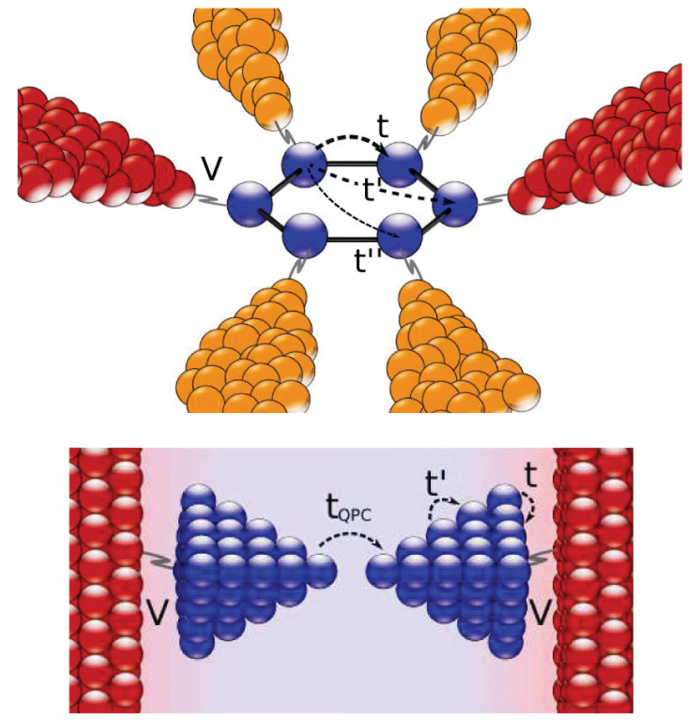

FIG. 1 (color). Abstract scheme of the nanosystems investigated: benzene geometry (top) and quantum point contact with 110 atoms (bottom). The individual nano-objects (blue sites) are connected by tunneling channels with amplitude $t$. These amplitudes might be rather complex and dependent on the distance as exemplified by the $t^{\prime}$ and $t^{\prime \prime}$ tunneling channels. The whole nano system is connected via some additional tunneling channels $V$ to the environment sketched schematically in red and orange and assumed to be noninteracting. Possible experimental realizations are coupled quantum dots or molecules connected to source and drain electrodes or a cluster of Fe atoms with the environment being a surface instead of the indicated geometry. Electronic correlations are particularly strong because of the nanostructuring. quantization operators and orbital matrices in the Green functions and self-energies below.

As an exact solution of Hamiltonian (1) is possible at most for $O(10)$ interacting sites, we here propose an approximate DГA solution. To this end, we first need to calculate the fully irreducible $n$-particle vertex on every site $i$ with the interacting Green function $G_{i i}(\omega)$ and Coulomb interaction $U_{i}$ as an input, see Fig. 2. In practice, this is done by numerically calculating the corresponding $n$-particle vertex of an associated AIM. Note that the effort for this computationally most expensive step only grows linearly with the number of sites and is easily parallelizable. From the $n$-particle vertex in turn, we recalculate the Green function and proceed with the first step until convergence. In the case $n=1$, the one-particle fully irreducible vertex is simply the self-energy $\Sigma(\omega)$ which is directly related to $G_{i i}(\omega)$ through the Dyson equation given in Fig. 2 in matrix notation for the site indices $i j$. For $n=$ 2 , one needs instead to use the parquet equations to go from the irreducible vertex to the reducible one and the exact equation of motion to get the self-energy before proceeding with the Dyson equation, similarly as discussed in Ref. [27] for an infinite system.

Let us note that the approach becomes exact in several limits: (i) $U \rightarrow 0$, (ii) $V \rightarrow \infty$, (iii) number of connections to neighbor sites $Z \rightarrow \infty$, and, if each site couples to its own lead (iv) $t \rightarrow 0$. While the exact QMC simulation is impossible for large clusters due to the so-called sign problem, our method is sign problem free. For $n=2$ the approach also takes into account the Cooperon diagrams so that weak localization physics is explicitly included, as are spin fluctuations.

Validation vs exact result.-As a first test case and to validate the approach for $n=1$ against the numerically exact solution, we consider the six-site benzene geometry of Fig. 1 (upper panel) with a constant density of states $\rho$ in the contacts from $-D$ to $D$ around the Fermi level $(D=$ $2 t, t=1$ sets our unit of energy in the following); and a site-diagonal hybridization $V_{i \nu k}=V \delta_{i \nu}$. Two topologies are considered: (i) hopping $t_{i j}$ restricted to the two nearest

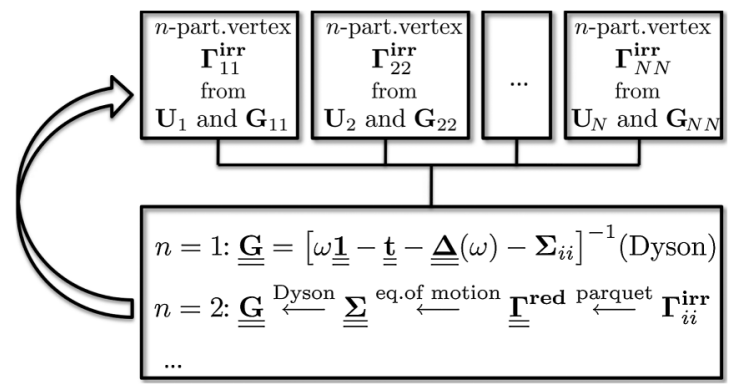

FIG. 2. Nano-DГA algorithm consisting of two selfconsistency steps: (1) from the local Green function $G_{i i}$ and Coulomb interaction $U_{i}$ the $n$-particle fully irreducible vertex $\Gamma_{i i}^{\mathrm{irr}}$ has to be calculated; (2) from $\Gamma_{i i}^{\mathrm{irr}}$ a new Green function $G_{i i}$ is determined through the Dyson and parquet equation, respectively. 
neighbors in the hexagon (NN $t$ ) and (ii) an equal hopping to all sites (all $t$ ). Both DГA and the exact solution are calculated by means of QMC simulations [41] for $U=5 t$, temperature $T=0.05 t$.

Figure 3 shows the calculated local spectral function in an interval $2 T$ around the Fermi level (set to zero) $\overline{A(0)}=$ $\int d \omega A(\omega) /\left[e^{\omega / 2 T}+e^{-\omega / 2 T}\right]$. The results clearly show that nano-DГA is reliable both for a hybridization $V \gtrsim t$ and if enough neighbors (in our case 5 in the all $t$ topology) are involved in the hopping processes. There are some deviations for the NN $t$ case if $V \lesssim t$ since in this situation nonlocal correlations such as those involved in forming a two-site singlet are relevant. If the hopping is to all neighbors though, no deviations could be identified all the way down to the last calculable point $V=0.8 t$. Below $V=$ $0.8 t$, the sign problem becomes too severe [average sign $O\left(10^{-3}\right)$ ], and only the nano-DГA solution is possible-in a situation very favorable for nano-DГA because of the many neighbors. Also other approaches such as exact diagonalization are not possible since together with a modeling of the leads there are too many sites. Note that another indication for the small correction is the offdiagonal exact self-energy, which is smaller than $10^{-2} t$ for $V>t$ and nearest-neighbor hopping as well as for all $V$ and hopping to all neighbors.

In the inset to Fig. 3, we present the conductance $G$ through the benzenelike nanostructure (upper panel of Fig. 1), from one side of the molecule to the opposite side, calculated along the lines of Ref. [42], i.e., without vertex corrections. As the local spectral function, the conductance again shows the reliability of the calculation already at the $n=1$ level, with discernible deviations from

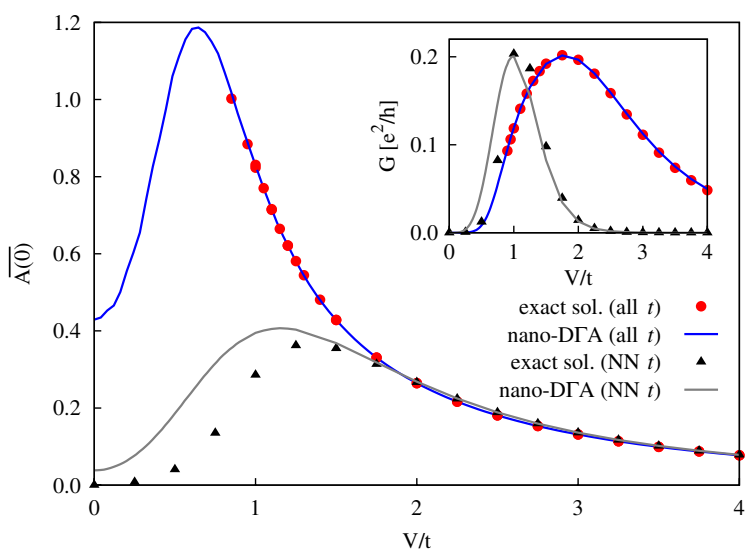

FIG. 3 (color online). On-site spectral function $\overline{A(0)}$ vs hybridization $V$, comparing nano-DГA (lines) with the exact QMC solution (symbols) for two different geometries: hopping to two nearest neighbors only and hopping to all neighbors. Inset: Conductance between two opposite leads of the benzene ring, i.e., between the dark red leads of Fig. 1, for both topologies of neighborhood. Together with the low off-site-diagonal selfenergy, the results show that nano-DГA is reliable if either the hybridization strength is sufficiently strong or if the hopping is to sufficiently many neighbors. the exact solution only for a low number of neighbors (i.e., two NN only) and small hybridization to the leads $V$. The results can be understood as follows: at small $V$, we have a conductance through two tunneling lead-benzene contacts, leading to an increase of $G \sim V^{4}$ (i.e., squared tunneling rate $\Gamma=\pi V^{2} \rho$ ). In the large $V$ region on the other hand, a Kondo resonance between the individual sites of the molecule and the respective lead forms, which suppresses the competing intermolecule hopping and hence the transport through it. If hopping between all sites is allowed (all $t$ ), this effect is less pronounced since there is a direct hopping channel between the opposite sites to which the voltage has been applied. Experimentally, transport through a benzene ring coupled to two Pt leads has been measured recently [43]. For our geometry similarly large conductances of $O(0.1) e^{2} / h$ were observed.

Quantum point contact $(Q P C)$.- - To demonstrate the suitability of the approach for more complex nanosystems, let us now consider a QPC. Experimentally it can be realized, e.g., through a mechanically controllable break junction of a conducting wire, see, e.g., Refs. $[44,45]$ and, with a molecule in between, Ref. [46]. The assumed geometry is based on a body-centered cubic basic structure narrowed to a double-cone-like junctions as shown in Fig. 1 (lower panel). For the moment we assume a single band which might be realizable in more complex wires such as cuprate and cobaltate wires, but realistic calculations, e.g., in the spirit of LDA + DMFT [33-36], are certainly possible. The parameters are $U=4 t, t=-1$, $t^{\prime}=-0.4, V=0.4$, and $T=0.05 t$. Each calculation takes about $10 \mathrm{~h}$ with a mild parallelization on 25 Nehalem Intel processor cores $(\mathrm{X} 5550,2.66 \mathrm{GHz})$, showing that much bigger calculations or calculations with orbital realism are possible. When slowly breaking up the junction the hybridization (tunneling) $t_{\mathrm{QPC}}$ between the two atoms form-

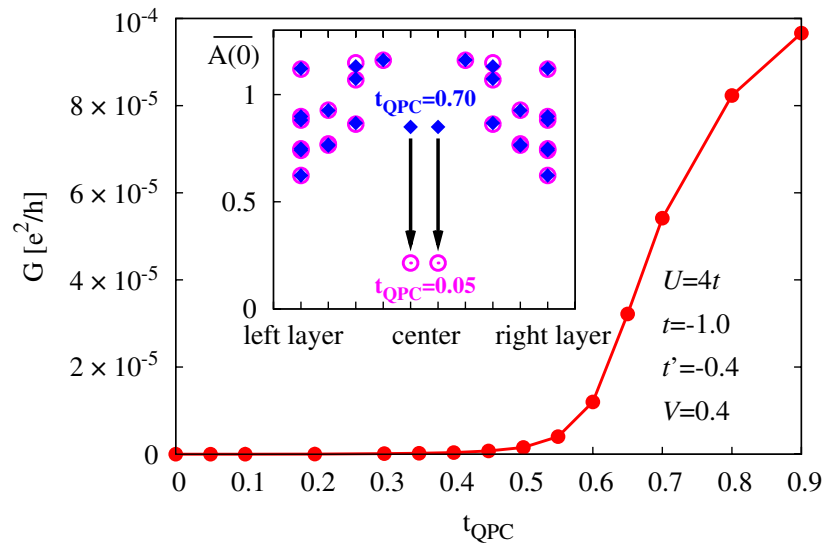

FIG. 4 (color online). Conductance $G$ across the quantum point contact (QPC) of lower panel of Fig. 1 vs hybridization $t_{\mathrm{QCP}}$ between the two atoms of the QPC. $G$ becomes almost zero for $\left|t_{\mathrm{QCP}}\right| \lesssim 0.5 t$. Inset: Spectral function layer by layer across the quantum point contact for two $t_{\mathrm{QCP}}$ 's below and above the conductance increase, revealing a local Mott-Hubbard transition in the two atoms forming the QPC. 
ing the point contact will change most strongly and is hence varied.

Surprisingly, we observe a dramatic reduction of the conductance for $\left|t_{\mathrm{QPC}}\right| \lesssim 0.5 t$ in Fig. 4. Breaking up the junction triggers a local Mott-Hubbard "transition" (more precisely a crossover) of the two atoms forming the quantum point contact, see Fig. 4 inset. Therefore the conductance drop with increasing distance between the two atoms is faster than the exponential decay of $t_{\mathrm{QCP}}$ with distance. Our findings might explain similar experimental observations [47] in transition-metal point contacts with partially filled $d$ shells, where the electrons are actually similarly strongly correlated as in our calculation. This effect could not have been revealed with hitherto employed methods [45] such as LDA, Landauer formalism, or Coulomb blockade calculations.

We thank A. Georges, P. Hansmann, D. Jacob, A. Katanin, S. Lichtenstein, and S. Okamoto for discussions. This work has been supported in part by the Austrian Science Fund (FWF) through science college WK004 (A. V.) and by the National Science Foundation under Grant No. PHY05-51164. Calculations have been performed on the Vienna Scientific Cluster.

[1] C. W. Beenakker, Phys. Rev. B 44, 1646 (1991).

[2] D. Goldhaber-Gordon et al., Nature (London) 391, 156 (1998).

[3] S. M. Cronenwett, T.H. Oosterkamp, and L.P. Kouwenhoven, Science 281, 540 (1998).

[4] J. Schmid et al., Physica (Amsterdam) 256B-258B, 182 (1998).

[5] L. Kouwenhoven and L. Glazman, Phys. World 14, 33 (2001).

[6] W. G. van der Wiel et al., Rev. Mod. Phys. 75, 1 (2002).

[7] A. Hübel et al., Phys. Rev. Lett. 101, 186804 (2008).

[8] J. K. Gimzewski, E. Stoll, and R. R. Schlittler, Surf. Sci. 181, 267 (1987).

[9] C. Joachim, J. K. Gimzewski, and A. Aviram, Nature (London) 408, 541 (2000).

[10] M. H. Hettler et al., Phys. Rev. Lett. 90, 076805 (2003).

[11] I. M. L. Billas et al., Phys. Rev. Lett. 71, 4067 (1993).

[12] V. Madhavan et al., Science 280, 567 (1998).

[13] J. Li et al., Phys. Rev. Lett. 80, 2893 (1998).

[14] M. Ternes, A. J. Heinrich, and W.-D. Schneider, J. Phys. Condens. Matter 21, 053001 (2009).
[15] T. A. Costi et al., Phys. Rev. Lett. 102, 056802 (2009).

[16] P. W. Anderson, in Moment Formation In Solids, edited by W. Buyers (Plenum Press, New York, 1984), p. 313.

[17] A. C. Hewson, The Kondo Problem to Heavy Fermions (Cambridge University Press, Cambridge, 1993).

[18] R. Bulla, T. A. Costi, and T. Pruschke, Rev. Mod. Phys. 80, 395 (2008).

[19] M. Caffarel and W. Krauth, Phys. Rev. Lett. 72, 1545 (1994).

[20] E. Koch, G. Sangiovanni, and O. Gunnarsson, Phys. Rev. B 78, 115102 (2008).

[21] U. Schollwöck, Rev. Mod. Phys. 77, 259 (2005).

[22] T. Maier et al., Rev. Mod. Phys. 77, 1027 (2005).

[23] W. Metzner and D. Vollhardt, Phys. Rev. Lett. 62, 324 (1989).

[24] A. Georges and G. Kotliar, Phys. Rev. B 45, 6479 (1992).

[25] A. Georges et al., Rev. Mod. Phys. 68, 13 (1996).

[26] G. Kotliar and D. Vollhardt, Phys. Today 57, No. 3, 53 (2004).

[27] A. Toschi, A. A. Katanin, and K. Held, Phys. Rev. B 75, 045118 (2007).

[28] A. N. Rubtsov, M.I. Katsnelson, and A.I. Lichtenstein, Phys. Rev. B 77, 033101 (2008).

[29] M. Jarrell, Phys. Rev. Lett. 69, 168 (1992).

[30] K. Byczuk et al., Nature Phys. 3, 168 (2007).

[31] A. Macridin et al., Phys. Rev. Lett. 99, 237001 (2007).

[32] A. Toschi et al., Phys. Rev. Lett. 102, 076402 (2009).

[33] V. I. Anisimov et al., J. Phys. Condens. Matter 9, 7359 (1997).

[34] A. I. Lichtenstein and M. I. Katsnelson, Phys. Rev. B 57, 6884 (1998).

[35] G. Kotliar et al., Rev. Mod. Phys. 78, 865 (2006).

[36] K. Held, Adv. Phys. 56, 829 (2007).

[37] S. Florens, Phys. Rev. Lett. 99, 046402 (2007).

[38] Note two related extensions, the inhomogeneous DMFT [39] which deals with stacked layers and the real space DMFT [40] for cold atoms, without environment.

[39] M. Potthoff and W. Nolting, Phys. Rev. B 59, 2549 (1999); 60, 7834 (1999).

[40] M. Snoek et al., New J. Phys. 10, 093008 (2008).

[41] J.E. Hirsch and R. M. Fye, Phys. Rev. Lett. 56, 2521 (1986).

[42] A. Georges and Y. Meir, Phys. Rev. Lett. 82, 3508 (1999).

[43] M. Kiguchi et al., Phys. Rev. Lett. 101, 046801 (2008).

[44] E. Scheer et al., Nature (London) 394, 154 (1998).

[45] N. Agraït, A. L. Yeyati, and J. M. van Ruitenbeek, Phys. Rep. 377, 81 (2003).

[46] C. Kergueris et al., Phys. Rev. B 59, 12505 (1999).

[47] J. M. Krans et al., Phys. Rev. B 48, 14721 (1993). 